\title{
TREAT Modeling and Simulation Strategy
}

Mark D. DeHart

Idaho National Laboratory

September 2015

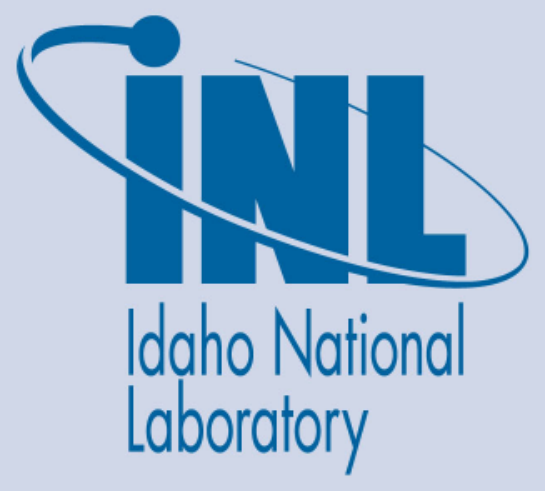

The INL is a U.S. Department of Energy National Laboratory operated by Battelle Energy Alliance 


\section{DISCLAIMER}

This information was prepared as an account of work sponsored by an agency of the U.S. Government. Neither the U.S. Government nor any agency thereof, nor any of their employees, makes any warranty, expressed or implied, or assumes any legal liability or responsibility for the accuracy, completeness, or usefulness, of any information, apparatus, product, or process disclosed, or represents that its use would not infringe privately owned rights. References herein to any specific commercial product, process, or service by trade name, trade mark, manufacturer, or otherwise, does not necessarily constitute or imply its endorsement, recommendation, or favoring by the U.S. Government or any agency thereof. The views and opinions of authors expressed herein do not necessarily state or reflect those of the U.S. Government or any agency thereof. 


\title{
TREAT Modeling and Simulation Strategy
}

\author{
Mark D. DeHart \\ Idaho National Laboratory
}

September 2015

Idaho National Laboratory

Reactor Physics Analysis and Design Dept.

Idaho Falls, Idaho $\mathbf{8 3 4 1 5}$

http://www.inl.gov

\author{
Prepared for the \\ U.S. Department of Energy \\ Office of Nuclear Energy \\ Under DOE Idaho Operations Office \\ Contract DE-AC07-05ID14517
}




\title{
TREAT Modeling and Simulation Strategy
}

\author{
Mark D. DeHart \\ Idaho National Laboratory
}

\begin{abstract}
This report summarizes a four-phase process used to describe the strategy in developing modeling and simulation software for the Transient Reactor Test Facility. The four phases of this research and development task are identified as (1) full core transient calculations with feedback, (2) experiment modeling, (3) full core plus experiment simulation and (4) quality assurance. The document describes the four phases, the relationship between these research phases, and anticipated needs within each phase.
\end{abstract}

\subsection{INTRODUCTION}

Operating from February 1959 until April 1994, the Transient Reactor Test Facility (TREAT) at Idaho National Laboratory (INL) was designed and built to be able to conduct transient tests on reactor materials. In such TREAT tests, the material would be subjected to neutron pulses that could simulate reactor accident conditions ranging from mild transients (e.g., anticipated transient without scram, or ATWS) to severe reactor accidents, such as a reactivity insertion accident (RIA). TREAT was originally constructed to test fast reactor fuels but has also been used for light water reactor fuel testing as well as other exotic special purpose fuels (i.e. space reactors).

TREAT is an air-cooled, thermal spectrum test facility designed to evaluate reactor fuels and structural materials, by inducing intense fission heating in the nuclear fuel being tested, to test nuclear reactor fuels under severe reactor-accident conditions, and to provide non-destructive test data through neutron radiography of fuel samples. As such, TREAT was used to study fuel meltdowns, metal-water reactions, interactions between overheated fuel and coolant, and the transient behavior of fuels for high-temperature systems. The open core design of TREAT also allowed for the detailed monitoring of experiments during a test. TREAT was specifically designed to test prototypic-sized reactor fuel pins and bundles under transient power conditions. During its 35 years of operation, it conducted thousands of transient experiments.

However, during its operating history, although computational methods improved, they never proved adequate for accurate core modeling. Three-dimensional Monte Carlo (KENO-IV) calculations were used to understand the shape of the core power distribution with various rod positions and likely used to confirm rod worth measurements. However, the transient portion of operations was never especially accurate, requiring a number of "calibration" tests to justify adjustment of point kinetics simulations of the transient to correct for power prediction in the experiment vehicle. Even then, the peak power deposition prediction was often in error by $+/-10 \%$ [1]. Note that the calibration tuning approach was 
used to obtain the desired power deposition in the experiment, but that this did not provide useful information on the core power distribution itself during a transient.

TREAT operated in a different environment than exists today, both in terms of requirements to operate and the cost of operation. Reactor analysis methods have evolved to the point that there is generally a high degree of confidence in calculations that are used for operations. Commercial reactors desire to optimize their fuel management approaches to reduce operating costs. They have heavily invested in computational methods to improve reactor modeling. National laboratories and university research are constantly pushing the state-of-the-art forward, developing method that are generally adopted first by the U.S. Department of Energy (DOE) and Nuclear Regulatory Commission (NRC), and when appropriate adopted or adapted for industrial needs. However, methods for rigorous prediction of reactor behavior under transient conditions have not been pursued. Kinetics phenomena are rarely an issue for normal operations, and basic point kinetics approaches may be conservatively applied in safety analysis for performance and licensing concerns. Thus an accurate and comprehensive kinetics toolset that can be used for TREAT transient modeling is not readily available.

The requirements that will be imposed for TREAT operation have not been fully developed, but it is known that the lifetime of the core will be limited by oxidation/degradation of the Zircaloy- 3 cladding used on all fuel and a subset of reflector elements. To minimize oxidation, an administrative limit for maximum clad temperature of $600 \mathrm{C}$ has been set. This temperature limit can impose significant restrictions on transient power pulses after uncertainties are accounted for. In addition, the operational mode of TREAT in the future will most likely not have the budget available for a series of calibration transients for each new experimental campaign. Hence there is strong motivation to have a high fidelity modeling and simulation tool in place for design of experiments and for estimating experiment responses to a given transient. Further, it will be important to be able to closely predict power deposition in an experiment for a given transient pulse; the investment of several days to perform pre-transient calibration may be prohibitively expensive. Hence, the Nuclear Engineering Advanced Modeling and Simulation (NEAMS) program has committed to fund efforts to development of appropriate modeling and simulation capabilities for TREAT and TREAT experiments.

The purpose of this report is to describe the strategy being employed at INL for development and deployment of an advanced yet practical simulation tool that can predict the response of an experiment design to a transient pulse in TREAT. This tool must be practical in the sense that it must be verified, validated and usable by TREAT operations and experiment analysts. In addition, the performance of the tool must be evaluated to determine the key aspects of the physics of both experiment and core that are most sensitive to modeling assumptions and approximations.

The tool selected for this work was the MAMMOTH multi-physics code for reactor analysis [2]. The following section provides an overview of MAMMOTH functionality. The subsequent sections then describe a four-phase strategy developed to completely adapt methods and capabilities in MAMMOTH to TREAT analysis for both core transients and coupled experiment performance.

\subsection{METHODS}

INL has developed high fidelity, tightly coupled multi-physics modeling under the Multi-physics Object Oriented Simulation Environment (MOOSE) framework [3]. The MOOSE framework consists of several 
consistently designed, pluggable interfaces that scientists and engineers may use to solve domain-specific problems. Internally, MOOSE utilizes the finite element method mathematical modeling technique due to its generality and wide applicability. In this work, the MOOSE-based reactor physics tool MAMMOTH was employed to perform coupled calculations with several relevant physics modules. MAMMOTH allows the coupling of a number of other MOOSE-based sub-applications including: Rattlesnake [4] for neutron transport, RELAP-7 [5] for low-resolution thermal-fluids, and BISON [6] for fuel performance analyses. In general MAMMOTH has great flexibility to solve complex reactor multi-physics problems. One approach is by solving a large system of interlinked nonlinear equations on the same mesh. These equations can be simultaneously solved with the Jacobian-Free Newton-Krylov (JFNK) method. However, because of needs in some problems for solutions on varying time scales, implicit coupling is not always optimal. Hence, a split operator approach is available where each sub-application solves its individual physics and has a coincident mesh with the other application [7,8]. Data is then shared between each sub-application coupling them using the MOOSE MultiApp system [7]. For this form of coupling an additional outer iteration and time substeps can be applied to make the physics more consistent. The MOOSE framework provides the necessary flexibility to perform multi-scale modeling where necessary, which will be imperative in experiment analysis and design. The general long-term technical objective of the currently work is to develop a set of high-resolution reactor physics and fuels performance models that can accurately predict the transient behavior of an in-core experiment when driven by a reactor transient.

A key feature of MOOSE-based applications is that a particular tool (in this case MAMMOTH) is compiled as a single executable code containing executable libraries for each component sub-application. In other words, within the MAMMOTH executable are embedded the full set of capabilities of BISON, Rattlesnake, RELAP-7 and MOOSE (among others, e.g., PRONGHORN and RAVEN). Communications between packages is done in-core using shared memory managed by MOOSE algorithms. MAMMOTH also inherits the advances in each of the applications and enforces code compatibility for all applications. In the near future, additional sub-applications will be added to MAMMOTH to expand its range of capabilities. Although studies have been completed [9], this form of implicitly and explicitly coupled multi-physics capability existing in one code with a unified data/communications/solution structure is not known to exist for traditional reactor analysis applications [10].

The application of MAMMOTH to TREAT analysis is summarized in Ref. 2. A report detailing research and development completed in FY15 is provided in Ref. 11.

\subsection{OVERVIEW OF STRATEGY}

This strategy has been developed based on (a) existing MAMMOTH capabilities and known weaknesses, (b) familiarity with TREAT design and historical operations (c) anticipated requirements for future TREAT operations and (d) preliminary work completed to date. The development and deployment strategy for TREAT modeling and simulation has been conceived and divided into four phases:

I - Full Core Transient Calculations with Feedback,

II - Experiment Modeling,

III - Full Core + Experiment Simulation,

IV - Quality Assurance. 
Figure 1 illustrates the interconnectivity of these phases. Phases I and II are largely independent - the same tools are being applied for each Phase (MAMMOTH and its constituent animals), but the analysis end of the work is independent and can be performed in parallel. However, although Phase III is likely to start before Phases I and II are completed, it will build on, and in fact rely on, the successes of the earlier work. Finally, Phase IV, quality assurance, has been a process within MOOSE and will continue through and beyond this project.

The following sections detail aspects of each phase that are anticipated to be required to guide this research to successful completion. These details are based on the current understanding of TREAT performance. Phases I and II are more well defined based on work in progress. Phase III represents the aspects anticipated to be necessary for successful completion of fully coupled core transient and experiment performance calculations. More details on requirements will be determined as this work proceeds, but of course cannot be predicted at this point based on our current level of knowledge.

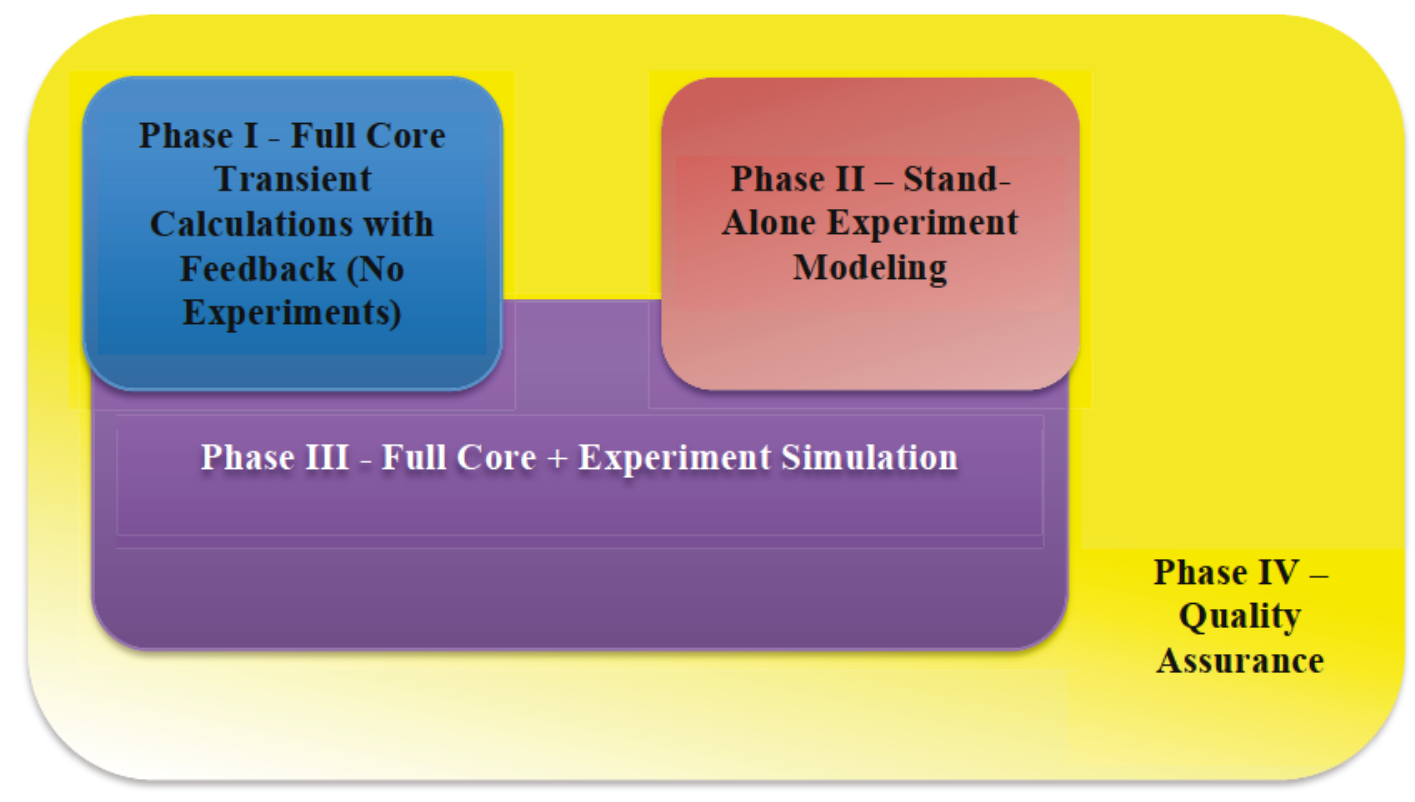

Fig. 1. Four Phased Approach for TREAT Modeling and Simulation.

\subsection{PHASE I - FULL CORE TRANSIENT CALCULATIONS WITH FEEDBACK}

Phase I provides the foundation for TREAT simulations. It is comprised of a ground-up study of TREAT physics to determine key aspects for core modeling, without considering the additional detail of an in-core experiment. It will also provide an opportunity for early validation of MAMMOTH calculations with and without thermal feedback. Finally, it is anticipated that this effort will identify shortcomings or inefficiencies in solution algorithms, which must be addressed to meet expected performance requirements. The following subsections detail issues that will need to be evaluated and addressed as appropriate to complete phase of TREAT modeling and simulation development. 


\subsection{Development of a Meshed Core Model}

Because both the BISON and Rattlesnake solution algorithms within MAMMOTH are in fact drawn from the finite-element framework of MOOSE, an appropriate mesh for finite element calculations will be needed. At present, most meshing work at INL is performed using CUBIT [13]. This is a powerful tool with scripting capabilities; specifications of mesh parameters and material assignments can be provided within the script to be able to rapidly generate meshes with different properties. Subsequent work, described later, will use steady state and transient simulations to determine minimum meshing requirements for spatial convergence of a solution.

\subsection{Kinetics Validation}

Because TREAT operates in a pulsed power manner for transient performance, an accurate simulation will rely on the accuracy of kinetics calculations within the Rattlesnake time-dependent neutron transport solver inside MAMMOTH. This work will start with basic verification of the time dependent solution of Rattlesnake for an infinite medium problem relative to point kinetics solutions. Computational benchmarks for simple, prescribed 2D and 3D configurations (Benchmarks 16-A1 and 16-A2 of Ref 12) will be analyzed using MAMMOTH. Once this has been completed, code-to-code testing is planned using an independent 3D kinetics solver with an Improved Quasi-Static (IQS) time integration scheme without thermal feedback. Ultimately comparison of a TREAT model to a documented TREAT transient will provide final validation, within the limits of the uncertainties in historical measurements.

\subsection{Cross-Section Testing}

Cross sections will be an integral facet of initial TREAT core simulations. Not only will neutron cross sections govern the calculation of initial and transient neutron distributions, changes in cross sections as a function of temperature will be used to capture and simulate the reactivity feedback that occurs in actual TREAT transients. The Rattlesnake solver within MAMMOTH requires appropriately weighted multigroup cross sections as a function of both temperature and space, to capture changes in spectrum in space and time. Appropriate methods to generate these weighted cross sections must be evaluated to determine the spatial effects as a function of temperature. It appears likely that the small size of TREAT relative to the mean free path of neutrons in graphite will require a three dimensional cross-section weighting approach, based on fluxes generated using a Monte Carlo approach.

Rattlesnake supports discrete ordinates, spherical harmonics and diffusion solutions; cross sections for each solution mode should be able to be generated and be shown to be consistent. Spatial homogenization effects on cross sections will also require study; such homogenization will provide a means to remove unimportant details from the core model. Appropriate homogenization from an initially explicitly meshed representation can be used to improve computational efficiency for core simulations.

\subsection{Criticality Validation}

The first test of cross sections will be in performing steady state analysis. Cross sections will be tested for various levels of homogenization and energy structures to determine requirements for meeting a reference Monte Carlo solution - both for the solution eigenvalue and spatial flux distributions. Materials should be representative of the TREAT core, but may start with an infinite medium of fuel material, 
moving to an infinite lattice of fuel elements, before moving to a full core model. Sufficient measured critical configurations for TREAT are available that may be used for validation of a full core model.

\subsection{Feedback Calculations}

Once confidence has been gained in generating cross sections evaluated as a function of temperature, the temperature interpolation approach used in MAMMOTH can be evaluated relative to a reference solution using interpolated values from pre-generated and tabulated reference temperatures.

The next step will to begin calculations in which Rattlesnake and BISON are linked as coupled solvers (implicitly and/or explicitly coupled with a Picard iteration approach). This should also start with simple configurations that can be used to understand the dynamics of Rattlesnake/BISON coupling. This again can begin with an infinite medium problem, and progress to the infinite lattice configuration used earlier. At this point, we will have moved beyond the ability to perform code-to-code comparison for validation, as such coupled transient calculations with thermal feedback are not available elsewhere. Evaluation at this point will be limited to an engineering assessment of the reasonableness of a solution, in as much as it matches the expected behavior of TREAT based on measured data. When the approach is finally evaluated based on a full core configuration for which measured data is available, it will become possible to more rigorously evaluate the transient simulation capabilities of MAMMOTH.

\subsection{Investigation of Feedback Properties and Physics}

In tandem with feedback calculations, it will be necessary to identify the thermal properties of the fuel and reflector (heat capacity and conductivity) as a value of temperature. It is already known that both of these properties has a temperature dependence - a functional form of those properties must be added to the core model for graphite fuel as a minimum. Initial work will likely assume that fuel regions within fuel elements can be assumed to be adiabatic and that heat transfer to clad or between elements is negligible during the first few seconds of a transient. More detailed thermal calculations will need to be performed to evaluate the validity of that assumption.

\subsection{Develop Understanding of Core Behavior During Transient Pulses}

With a working model, it will become possible to start operating and instrumenting a virtual core. This will let modelers begin to understand the physics of the core during steady-state and transient operation, such as leakages, spatial flux and reaction rate distributions, control rod effects, coupling between rod sets, variation of kinetic parameters as a function of temperature and rod motion, etc. Many of the assumptions and simplifications that may be applied to a core model may be driven or limited by the physics of the system. For example, axial leakage in air channels already identified in early work will require directionally dependent diffusion coefficients or higher order spherical harmonics or quadrature sets to compensate for the strong angular variation in fluxes.

\subsection{Code Efficiency and Bug Fixes}

Progress in each of the above areas are expected to uncover bugs, code bottlenecks, and inefficiencies that become manifest as larger and more complex problems are studied. These issues cannot be predicted but certainly can be expected as part of the development process. 


\subsection{Input Automation}

As analyses become more proceduralized, calculations will be enhanced by automating model development. This was initiated in the development of mesh scripts, to allow "core loading" by script inputs. To improve usability, a front-end interface will ultimately be of value to simplify model and mesh develop processes. Automated generation of cross sections sets for a given core configuration would follow from this. And as experience is developed in transient input for code coupling, it will be possible to modify input processors to allow default values that no longer need be input other than to override a given default.

\subsection{PHASE II - EXPERIMENT MODELING}

Phase II will be essentially independent of Phase I and can be performed on parallel. This phase of modeling and simulation will begin the process of simulation of configurations that resemble anticipated experiment configurations. Initial work will focus on pin-level simulations to study the coupling process, before moving to meshing and simulation of full experiment rig configurations. However, the history of LWR pin simulations with MOOSE-based tools is much more mature than reactor kinetics, and this phase is anticipated to require less development than the core modeling effort. The following subsections will describe the steps expected in this work.

\subsection{Evaluation of Fuel Performance/Thermal-Hydraulics Coupling for Postulated Transients}

Initial research will be on simple coupled BISON/RELAP-7 calculations for fuel pins in simulated accident scenarios. This will focus on the dynamics of flow calculations and how they relate to fuel pin and corresponding mesh restructuring with burnup. BISON provides internal burnup predictions based on a generalized fuel pin model.

\subsection{Evaluation of full Neutron Transport/Fuel Performance/Thermal-Hydraulics coupling for postulated transients}

Full coupling between Rattlesnake, BISON and RELAP-7 will be essential for modeling experiment performance under transient scenarios. Although TREAT transients will span very little exposure time and depletion calculations will not be performed, many transient experiments are expected to be performed on highly burned fuel. Depletion calculations will be performed in a fully-coupled environment to obtain a representative fuel performance simulation. The resulting fuel configuration will then be moved into a transient test configuration. Hence, this work will focus on a fuel cycle burn simulation followed by an accident simulation in a single calculation to improve the understanding of the mechanisms involved in both phases.

\subsection{Improved Code Efficiency}

Modeling deficiencies or weaknesses are known to exist in the process described above. The implementation of three way coupling using Picard iterations in MAMMOTH is limited and has been found to result in numerical instabilities resulting in small time step requirements. This is related to algorithms that exist within MOOSE, and are being addressed by the MOOSE development team. Other 
lesser issues are also being tackled. These are expected to result in significantly improved code performance. In addition, while MOOSE supports restart of single physics and implicitly coupled multiphysics calculations, full restart of explicitly coupled Picard iterations is not well supported. MOOSE development seeks to address this issue, to allow separation of a long-term burnup calculation from a subsequent rapid transient, while retaining fuel/clad structure evolution and related mesh adaptation from the end of the former to use as a starting point for the latter. These know issues will no doubt be succeeded by new issues uncovered as development efforts proceed.

\subsection{Develop an Understanding of the Effect of Coupling and Mechanical Feedback}

As with TREAT core modeling simulations, it will become important to begin to understand the physics of fuel performance and flow behavior in (1) an extended neutron driven burnup cycle, (2) a decay heat driven post-scram transient (with flow, flow reduction, and flow blockage or loss), and of course (3) rapid power excursions (also with and without flow). The coupled interactions between these different physics phenomena are known to be complex and expected to be challenging. Extensive testing and simulation will be crucial to be able develop a confidence in modeling and understanding of simulation responses.

\subsection{Benchmark Against Experiments and Code-to-Code Comparisons}

A number of multi-physics benchmarks are currently being evaluated through national and international programs including OECD Nuclear Energy Agency and IAEA benchmark sets and expert groups. INL has already engaged in a few of these collaborative efforts and will continue to take advantage of expert collaboration to improve benchmarking, identify weaknesses and areas for modeling focus. These opportunities are expected to increase with increasing international interest in multi-physics simulations.

\subsection{Improved User Interface}

Again, as with the core modeling efforts, experience in model development and needs are expected to provide a basis for streamlining model development and to develop a more user-friendly environment. Interaction with analysts and user feedback will be used to improve the modeling interfaces for users.

\subsection{Simulation of Historical Experiments with Externally Measured or Calculated Power Sources}

The first step toward fully coupled core/experiment simulations is anticipated to be manifested by introduction of a simulated transient represented as a time-dependent flux boundary condition. Measured power transient data and/or fluxes calculated by standalone core transient simulations will be used as a transient boundary condition to allow computational efforts to focus on experiment modeling alone. This will also provide a benchmark of sorts for subsequent fully coupled analyses.

\subsection{PHASE III - FULL CORE + EXPERIMENT SIMULATION}

Building on successful deployment of analysis methods for both core and experiment simulation, the final development phase in this project will be to combine the two capabilities to fully simulate TREAT experiment testing. Much research and development is anticipated to be able to get to this point, but if Phases I and II are executed successfully and as planned, Phase III will be primarily focused on any 
remaining integration issues, and then will turn to validation and testing. As before, these subsections will briefly describe the strategy to fully test, validate and deploy TREAT modeling and simulation capabilities.

\subsection{Integration of Core and Experiment Simulators}

If implemented and tested as planned, with continued MOOSE framework development, integration of the two simulation capabilities should be straightforward. However, code development rarely proceeds precisely as planned, and integration issues will be expected. Once any such issues are resolved, renewed optimization and implementation adjustments are planned, most likely within the MOOSE framework itself. The nature and scope of such improvements cannot be predicted and will be addressed as appropriate at that time.

\subsection{Simulation of Simple Experiments}

First full core simulations will start with simple targets. A large number of TREAT calibration measurements were performed with flux wires and single or sets of fresh fuel elements with no flow. Post-irradiation assay provided integrated reaction rate/flux in the samples. These configurations are simpler and can be simulated with reduced order multi-physics coupling in the experiment vessel.

\subsection{Simulation of Multi-Physics Experiments with Fluid Flow}

A number of experiments were performed with active fluid flow during the transient. The majority of these were with sodium, but for single-phase flow these would provide a configuration that could be modeled. At this point, it is not clear if sufficient data is available in the data to be able to validate multiphysics calculations. In that event, calculations will be performed simulating hypothetical LWR fuels with water moderation (single phase). Ongoing design of the Treat Water Environment Recirculating Loop (TWERL) will also be a likely candidate for comparison to other independent design work.

\subsection{Simulation of Multi-Physics Experiments with Phase Change}

Phase change and loss of coolant experiments were performed in earlier TREAT experiment series. However, it is not clear if experiments were performed with boiling water in a looped flow system, which is of most interest in current experiment designs. And, as above, it is not clear if sufficient data is available in the data to be able to validate multi-physics calculations. However, calculations will be preformed with hypothetical experiment designs, also likely based on TWERL, to ensure that results look reasonable.

\subsection{Demonstration of Multi-Scale Modeling}

In FY15 a three-year INL-internal Laboratory Directed Research and Development project focused on multi-scale modeling was completed. The intent of this research, based on mortar finite element method (MFEM) [14] was to provide the appropriate boundary coupling terms for combined high order/low order solutions. A portion of this work and its significance was discussed in an earlier NEAMS report [15]. This approach will continue development, and ultimately is hoped to allow a low order (i.e., diffusion) simulation of neutron transport in the TREAT core to drive a high order (i.e., discrete ordinates) solution 
within the experiment cavity during a transient. This will allow computational resources to be dedicated to a detailed solution within the experiment, but still provide a lower cost yet accurate solution for the time dependent neutron source resulting from a TREAT pulse.

\subsection{Engagement with Core Startup Operations for Recommendation and Development of Validation Tests.}

Perhaps one of the most important opportunities for experimental validation of MAMMOTH for TREAT physics will result from design and eventually startup testing for TREAT over the next 3-5 years. The NEAMS PI for TREAT modeling and simulation development regularly participates in TREAT operations planning and experiment design meetings and provides feedback on the status of MAMMOTH modeling and simulation research. All participants in these meetings, all the way to the top of the TREAT restart management team, are in agreement on the need for cooperative planning that will assist in future experiment design but will also provide an opportunity to for core/experiment modelers participate in data acquisition planned and deployment, to provide additional quality measurements of performance data in the form needed for validation.

\subsection{PHASE IV - QUALITY ASSURANCE}

The MAMMOTH development team and respective owners of constituent animals within MAMMOTH are actively engaged in implementation of an NQA-1 level quality assurance plan for software consistent with INL SQA requirements and those identified in NEAMS Software Quality Assurance (SQA) guidance in Ref. 16. The MOOSE development team is funding internal quality assurance experts and external consultants preparing MAMMOTH and its herd (BISON, RELAP-7, RAVEN and Rattlesnake) teams for NQA-1 assessments. BISON will undergo an NQA-1 assessment November 16-19, with a team led by Michael Lachner (member of ASME NQA-1 committee). RELAP-7 and RAVEN will be assessed March 28-31, 2016, with Rattlesnake and MAMMOTH undergoing assessment at the beginning of September 2016. Aspects of this implementation are detailed below.

\subsection{Verification and Validation}

Planned validation efforts have been described in previous sections and will not be repeated here. Verification efforts are an ongoing process, based on analytical solutions and repeated regression testing after every code change. An independent review and approval is required for each code change before it is formally committed to the MOOSE repository. A repository base on the GIT repository system [17] is used for source code control, testing and archival. Test problems are developed to test as much of the MOOSE repository as possible, with regression tests run each night and the status of code evaluations managed in real time using the MOOSE Build system [18] developed at INL for this purpose. A code coverage score of greater than $80 \%$ is required for code assessment - new capabilities require new code coverage tests to be included.

One of the most significant advantages of the MOOSE system is the fact that the solution algorithms and most auxiliary functions are derived from the MOOSE framework, including embedded PETSc [19] and libMesh [20] software that is externally maintained but upon which much functionality rests. Software $\mathrm{V} \& \mathrm{~V}$ for these packages is inherited by MOOSE-based software - it is not necessary to verify and validate embedded libraries if that level of quality assurance is performed in the maintenance of those 
packages. It is only necessary to verify proper implementation of calls to those packages and perform $\mathrm{V} \& \mathrm{~V}$ of code developed specifically for functions native to the parent software.

\subsection{Documentation}

Documentation of theory and user's manuals are a requirement of INL SQA and will be completed in preparation for respective assessments. An updated code management procedure is being considered to require a documentation update, or demonstration that no update is required, before software updates may be merged into the MOOSE base repository, although this in itself is not an SQA requirement.

\subsection{Transition to a Safety-Basis Software Package}

At present MAMMOTH QA is maintained at Quality Rigor Level (QRL) 3, as a basic research and development tool. This Quality Rigor Level will be required to evolve as the application of the software begins to be applied to TREAT systems. In order to be used for scoping studies of TREAT performance for operational needs or for the design of experimental rigs, MAMMOTH quality control procedures will be required to be updated to QRL 2. Ultimately, before in may be used as a TREAT operations tool, MAMMOTH quality-related processes and procedures must be updated to QL1. The details of this process are beyond the scope of this report and are describe in more detail in Ref. 16. We simply acknowledge that this will be a requirement for MAMMOTH quality assurance as usage transitions from R\&D to safety-basis applications.

\subsection{Training}

User training will also transition from the developmental nature of training employed by INL staff in MOOSE and MAMMOTH training workshops to a formal, reviewed and documented process that is closely linked to software documentation. Again, the nature of this evolution is beyond the scope of this report.

\subsection{Issue Tracking and User feedback}

A key aspect of software development under the principle of continuous improvement will be to provide a means for user feedback to document problems or needs, together with a tracking system that will allow status updates on open items and the ability to close items when fully resolved. At present the full MOOSE team uses an issues tracking log available within the INL GitLab repository system. This process meets all current quality needs and expected to continue to be the means for user/developer interaction with other team developers.

\subsection{CONCLUSIONS}

This report has described the current research and development strategy for TREAT modeling and simulation using the reactor multi-physic tool MAMMOTH. The strategy has been divided into four phases that will take the initial MAMMOTH tool and take it to a full deployment state by the end of the project. No timeline is proposed; because this is a research venture that seeks to provide a full core transient simulation capability coupled with modeling of experiments located in the core during a transient, there is significant uncertainty in exactly what will be required and how much time and effort 
will be needed to meet all requirements. This document provides only a strategy with key elements that are currently expected to be necessary for a successful completion. However, the strategy will be adjusted as needed as new knowledge is obtained over the course of this work.

\subsection{REFERENCES}

1. G. R. IMEL, Personal Communication, June 2015.

2. M. D. DEHART, F. N. GLEICHER, J. ORTENSI, A. L. ALBERTI and T. S. PALMER, "MultiPhysics Simulation of TREAT Kinetics using MAMMOTH," Accepted for publication, Trans. Am. Nucl. Soc., ANS Winter Meeting, Washington DC, November 8-12, 2015.

3. D. GASTON, C. NEWMAN, G. HANSEN, and D. LEBRUN-GRANDÉ, "MOOSE: A Parallel Computational Framework for Coupled Systems of Non-Linear Equations," Nucl. Eng. Design, 239, pp. 1768-1778 (2009).

4. Y. WANG. "Nonlinear Diffusion Acceleration for the Multigroup Transport Equation Discretized with $\mathrm{S}_{\mathrm{N}}$ and Continuous FEM With Rattlesnake," Proceedings to the International Conference on Mathematics, Computational Methods \& Reactor Physics (M\&C 2013), Sun Valley, Idaho, USA (May 2013).

5. D. ANDRS, et al., "RELAP-7 Level 2 Milestone Report: Demonstration of a Steady State Single Phase PWR Simulation with RELAP-7," INL/EXT-12-25924 (May 2012).

6. R. L. WILLIAMSON, et al., "Multidimensional Multi-physics Simulation of Nuclear Fuel Behavior," Journal of Nuclear Materials, 423: 149-163, (2012).

7. F. N. GLEICHER, et. al. "The Coupling of the Neutron Transport Application RATTLESNAKE to the Fuels Performance Application BISON," International Conference on Reactor Physics (PHYSOR 2014), Kyoto, Japan, (May 2014).

8. J. D. HALES et al., "Advanced multiphysics coupling for LWR fuel performance analysis," Annals of Nuclear Energy, 84: 98-110, (2015).

9. J. RAGUSA, and V.S. MAHADAVAN, "Consistent and accurate schemes for coupled neutronics thermal hydraulics reactor analysis," Nuclear Engineering and Design, 239: 566-579 (2009).

10. K. IVANOV and M. ACRAMOVA, "Challenges in coupled thermal-hydraulics and neutronics simulations for LWR safety analysis," Annals of Nuclear Energy, 34: 501-513 (2007).

11. J. ORTENSI, M. D. DEHART, F. N. GLEICHER, AND Y. WANG, "Full Core TREAT Kinetics Demonstration Using Rattlesnake/BISON Coupling Within MAMMOTH,” INL/EXT-15-36268, Idaho National Laboratory, August 2015.

12. ARGONNE COMPUTING CENTER. "Benchmark problem book." ANL- 7416, Suppl. 2 (1977).

13. R. MORRIS et al., "CUBIT 15.0 User Documentation," Sandia National Laboratory, https: //cubit.sandia.gov, (2015)

14. F. B. BELGACEM, "The mortar finite element method with Lagrange multipliers," Numer. Math. 84(2), 173-197, (Nov. 1999).

15. Y. WANG, M. D. DEHART, F. N. GLEICHER, J. ORTENSI AND S. SCHUNERT, "Year End Progress Report on Rattlesnake Improvements," INL/EXT-15-36639, Idaho National Laboratory, (Sept. 2015).

16. R. M. VERSLUIS, "NEAMS Verification and Validation Plan Requirements, Rev 0," U.S. Department of Energy, Office ofAdvanced Modeling and Simulation, (July 2013).

17. S. CHACON AND B. STRAUB, "Pro Git," $2^{\text {nd }}$ Edition, available online at <https://progit2.s3.amazonaws.com/en/2015-10-01-98c40/progit-en.848.pdf> (2014).

18. IDAHO NATIONAL LABORATORY, "MOOSE Build," https://www.moosebuild.com (2014). 
19. ARGONNE NATIONAL LABORATORY, "PETSc - Portable, Extensible Toolkit forScientific Computation," $<$ http://www.mcs.anl.gov/petsc/> (2015)

20. B. S. KIRK, J. W. PETERSON, R. H. STOGNER, AND G. F. CAREY, "libMesh: A C++ Library for Parallel Adaptive Mesh Refinement/Coarsening Simulations. Engineering with Computers, 22(34):237-254, (2006). 